\title{
Efficacy and safety of the application of extensive ablation in patients with atrioventricular re-entrant tachycardia (a retrospective study)
}

\author{
GuangZe $\mathrm{Xu}^{1}$, Zhikui Chen ${ }^{1}$, and Haiyan $\operatorname{Lin}^{1}$ \\ ${ }^{1}$ Li Huili Hospital
}

October 29, 2020

\begin{abstract}
Introduction: Radiofrequency catheter ablation (RFCA) has become the standard effective therapy for supraventricular tachycardias, but the reported success rates of ablation have differed across a large number of single-center studies. The main reason for tachycardia recurrence is accessory pathway (Ap)-mediated tachycardia, and the RFCA strategy may be related to recurrence. This study aims to compare the efficacy and safety of two different RFCA strategies for Ap-mediated tachycardia. Methods : We compared patients (group M) who underwent RFCA at extensive sites to patients (group S) with RFCA at a single site during the index procedure for Ap-mediated tachycardia. The efficacy and safety were assessed in the two groups. Results: A total of 882 patients with 898 Aps were enrolled in group S, and 830 patients with 843 Aps were enrolled in group M. The results showed that the cumulative numbers of recurrences (rates) at the 1st, 3rd, 6th, 12th, and 24th months after ablation were $4(0.5 \%)$ and $17(1.9 \%), \mathrm{p}<0.05 ; 5(0.6 \%)$ and $27(3.0 \%), \mathrm{p}<0.05 ; 6(0.7 \%)$ and $34(3.8 \%), \mathrm{p}<0.05 ; 6(0.7 \%)$ and $43(4.8 \%), \mathrm{p}<0.05$; and $7(0.8 \%)$ and $45(5.0 \%), \mathrm{p}<0.05$ in group $\mathrm{M}$ and group $\mathrm{S}$, respectively. Complications of chest pain, overactive vasovagal reaction, steam pop, and angina pectoris were rare in both groups. No valve damage, cardiac tamponade, or other serious adverse events occurred in either group. Conclusion: The strategy of performing extensive ablation reduced the recurrence rate and need for subsequent ablation of the Ap without increasing the risk of complications.
\end{abstract}

\section{Hosted file}

manuscript.pdf available at https://authorea.com/users/316179/articles/489689-efficacy-andsafety-of-the-application-of-extensive-ablation-in-patients-with-atrioventricular-reentrant-tachycardia-a-retrospective-study 